\title{
Exploration and Mapping Using the VFM Motion Planner
}

\author{
Santiago Garrido, Luis Moreno, and Dolores Blanco
}

\begin{abstract}
Efficient mapping of unknown environments is a fundamental function for mobile robot intelligence. To do so requires good exploration strategies and solving the simultaneous localization and mapping problem. The approach presented in this paper is an integration of our solutions into the problems of exploration and map building with a single robot. The exploration algorithm is based on the Voronoi fast marching (VFM) method to determine a motion plan toward the most unexplored and free zones of the environment. One consistent global map of the workspace is created using the simultaneous localization and modeling (SLAM) algorithm based on a nonlinear evolutive filter called the evolutive localization filter. The combination of the extended Voronoi transform and the fast marching method in the VFM method provides potential maps for robot navigation in previously unexplored dynamic environments. The logarithm of the extended Voronoi transform imitates the repulsive electric potential from walls and obstacles. The method uses a fast marching technique to determine a motion plan. A new strategy such that the robot determines the zones that it must explore in an autonomous way is described. As the robot carries out the exploration, it constructs a consistent map of the environment using the SLAM algorithm.
\end{abstract}

Index Terms-Exploration, mobile robots, robot mapping, simultaneous localization and modeling (SLAM).

\section{INTRODUCTION}

A UTONOMOUS exploration and mapping are fundamental problems to be solved as an autonomous robot carries out tasks in real unknown environments. Sensor-based exploration, motion planning, localization, and simultaneous mapping are processes that must be coordinated to achieve autonomous execution of tasks in unknown environments.

There is a variety of potential applications for autonomous mobile robots in such diverse areas as forestry, space, nuclear reactors, environmental disasters, industry, and offices. Potential tasks for autonomous mobile robots include maintenance, delivery, and security surveillance, which all require some form of intelligent navigational capabilities. A mobile robot will be a useful addition to these domains only when it can serve the following purposes: 1) robustly function under various environmental conditions; 2) operate without human intervention for long periods of time; and 3) provide some guarantee of task performance. The environments in which mobile robots must function are dynamic, unpredictable, and not completely

Manuscript received December 19, 2007; revised July 23, 2008. First published April 24, 2009; current version published July 17, 2009. The review of this paper was coordinated by Associate Editor Dr. Jesús Ureña.

The authors are with the Department of Systems Engineering and Automation, Universidad Carlos III de Madrid, 28911 Madrid, Spain (e-mail: sgarrido@ing.uc3m.es; moreno@ing.uc3m.es; dblanco@ing.uc3m.es).

Color versions of one or more of the figures in this paper are available online at http://ieeexplore.iee.org.

Digital Object Identifier 10.1109/TIM.2009.2016372 specifiable by a map beforehand. For the robot to successfully complete a set of tasks, it must dynamically adapt to changing environmental circumstances. Sensor-based planning makes use of the sensor-acquired information of the environment in its latest configuration and generates an adequate path toward the desired following position. Sensor-based discovery path planning is the guidance of an agent-a robot—without a complete a priori map by discovering and negotiating the environment to reach a goal location while avoiding all encountered obstacles. Sensor-based discovery (i.e., dynamic) path planning is problematic, because the path needs to continually be recomputed as new information is discovered.

To autonomously build a map of an unknown environment, this paper first presents an exploration and path-planning method based on the logarithm of the extended Voronoi transform (EVT) and the fast marching method. This path planner is called Voronoi fast marching (VFM) [7]. The EVT of an image gives a gray scale that is darker near the obstacles and walls and is lighter when far from them. The logarithm of the EVT imitates the repulsive electric potential in 2-D from walls and obstacles. This potential impels the robot far from obstacles. The fast marching method has been applied to path planning [34], and their trajectories are of minimal distance. They are not very safe, however, because the path is very close to obstacles, and more importantly, the path is not smooth enough. To improve the safety of the trajectories calculated by the fast marching method, avoiding unrealistic trajectories produced when the areas are narrower than the robot, objects and walls are enlarged in a security distance that assures that the robot does not collide and does not accept passages narrower than the robot's size.

The last step is to calculate the trajectory in the image generated by the logarithm of the EVT using the fast marching method. Then, the path obtained verifies the smoothness and safety considerations required for mobile robot path planning.

The advantages of this method include the ease of implementation, the speed of the method, and the quality of the trajectories. This method is used at a local scale that operates with sensor information (i.e., sensor-based planning).

To build the environment map while the robot carries out the exploration task, simultaneous localization and modeling (SLAM) is implemented. The algorithm is based on the stochastic search for solutions in the state space to the global localization problem through a differential evolution algorithm. This nonlinear evolutive filter, called evolutive localization filter (ELF) [20], stochastically searches along the state space for the best robot pose estimate. The set of pose solutions (the population) focuses on the most likely areas according to 
the perception and up-to-date motion information. The population evolves using the log-likelihood of each candidate pose according to the observation and the motion errors from the comparison between observed and predicted data from the probabilistic perception and motion model.

In the remainder of this paper, Section II reviews other approaches to spatial exploration and map learning. Section III presents our VFM motion planner. The SLAM algorithm is briefly described in Section IV. Then, Section V describes the specific exploration method proposed in this paper. Next, Section VI demonstrates the performance of the exploration strategy as it explores different environments according to the two possible ways of working for the exploration task. Finally, conclusions are summarized in Section VII.

\section{Previous and Related Work}

\section{A. Representations of the World}

Roughly speaking, there are two main forms for representing the spatial relations in an environment: 1) metric maps and 2) topological maps. Metric maps are characterized by a representation where the position of the obstacles is indicated by coordinates in a global frame of reference. Some of them represent the environment with point grids, defining regions that can be occupied or not by obstacles or goals [9], [19]. Topological maps represent the environment with graphs that connect landmarks or places with special features [12], [21]. In our approach, we choose the grid-based map to represent the environment. The clear advantage is that, with grids, we already have a discrete environment representation, which can readily be used in conjunction with the EVT function and fast marching method for path planning. The pioneer method for environment representation in a grid-based model was the certainty grid method developed at the Carnegie Mellon University [19] by Moravec and Elfes. He represents the environment as a 3-D or 2-D array of cells. Each cell stores the probability of the related region that is occupied. The uncertainty related to the position of objects is described in the grid as a spatial distribution of these probabilities within the occupancy grid. The larger the spatial uncertainty, the greater the number of cells occupied by the observed object. The update of these cells is performed during the navigation of the robot or through the exploration process by using an update rule function. Many researchers have proposed their own grid-based methods. The main difference among them is the function used to update the cell. Some of them are, e.g., fuzzy [25], Bayesian [8], heuristic probability [2], and Gaussian [3]. In histogramic in-motion mapping, each cell has a certainty value, which is updated whenever it is being observed by the robots sensors. The update is performed by increasing the certainty value by 3 (in the case of detection of an object) or by decreasing it by 1 (when no object is detected), where the certainty value is an integer between 0 and 15 .

\section{B. Approaches to Exploration}

This section relates some interesting techniques for exploratory mapping. They mix different localization methods, data structures, search strategies, and map representations.
Kuipers and Byun [13] proposed an approach to explore an environment and to represent it in a structure based on layers called spatial semantic hierarchy [12]. The algorithm defines distinctive places and paths, which are linked to form an environmental topological description. Then, a geometrical description is extracted. The traditional approaches focus on geometric description before the topological one. The distinctive places are defined by their properties, and the distinctive paths are defined by the twofold robot control strategy: 1) follow the midline or 2) follow the left wall. The algorithm uses a lookup table to keep information about the place visited and the direction taken. This allows a search in the environment for unvisited places. Lee [16] developed an approach based on Kuipers and Byun's work [13] on a real robot. This approach is successfully tested in indoor officelike spaces. This environment is relatively static during mapping. Lee's approach assumes that walls are parallel or perpendicular to each other. Furthermore, the system operates in a very simple environment with cardboard barriers. Mataric [21] proposed a map-learning method based on a subsumption architecture. Her approach models the world as a graph, where the nodes correspond to landmarks, and the edges indicate topological adjacencies. The landmarks are detected from the robot movement. The basic exploration process is wall-following combined with obstacle avoidance. Oriolo et al. [25] developed a grid-based environment mapping process that uses fuzzy logic to update the grid cells. The mapping process runs online [24], and the local maps are built from the data obtained by the sensors and are integrated into the environment map as the robot travels along the path defined by the $A^{*}$ algorithm to the goal. The algorithm has two phases. The first phase is the perception phase. The robot acquires data from the sensors and updates its environment map. The second phase is the planning phase. The planning module replans a new safe path to the goal from the new explored area. Thrun and Bucken [37], [38] developed an exploration system that integrates both evidence grids and topological maps. The integration of the two approaches has the advantage of disambiguating different positions through the grid-based representation and performing fast planning through the topological representation. Exploration is performed through the identification and generation of the shortest paths between unoccupied regions and the robot. This approach works well in dynamic environments, although the walls have to be flat and cannot form angles that differ more than $15^{\circ}$ from the perpendicular. Feder et al. [4] proposed a probabilistic approach to treat the concurrent mapping and localization using a sonar. This approach is an example of a feature-based approach. It uses the extended Kalman filter to estimate the localization of the robot. The essence of this approach is to take actions that maximize the total knowledge about the system in the presence of measurement and navigational uncertainties. This approach was successfully tested in wheeled land robot and autonomous underwater vehicles. Yamauchi [39], [40] developed the frontier-based exploration to build maps based on grids. This method uses a concept of frontier, which consists of boundaries that separate the explored free space from the unexplored space. When a frontier is explored, the algorithm detects the nearest unexplored frontier and attempts to navigate toward it by planning an obstacle-free 
path. The planner uses a depth-first search on the grid to reach that frontier. This process continues until all the frontiers are explored. Zelek [42] proposed a hybrid method that combines a local planner based on a harmonic function calculation in a restricted window with a global planning module that performs a search in a graph representation of the environment created from a computer-aided design map. The harmonic function module is employed to generate the best path, given the local conditions of the environment. The goal is projected by the global planner in the local windows to direct the robot. Recently, Prestes da Silva et al. [29] have investigated the performance of an algorithm for exploration based on the partial updates of a harmonic potential in an occupancy grid. They consider that as the robot moves, it carries along an activation window whose size is of the order of the sensors range.

Prestes da Silva et al. [30] propose an architecture for an autonomous mobile agent that explores while mapping a 2-D environment. The map is a discretized model for the localization of obstacles, on top of which a harmonic potential field is computed. The potential field serves as a fundamental link between the modeled (discrete) space and the real (continuous) space where the agent operates.

The proposed method in this paper can be included in the sensor-based global planner paradigm. It is a potential method, but it does not have the following typical problems of these methods enumerated by Koren-Borenstein [10]:

1) trap situations due to local minima (cyclic behavior);

2) no passage between closely spaced obstacles;

3) oscillations in the presence of obstacles;

4) oscillations in narrow passages.

The proposed method is conceptually close to the navigation functions of Rimon-Koditscheck [33], because the potential field has only one local minimum at the single goal point. This potential and the paths are smooth (similar to the repulsive potential function), and there are no degenerate critical points in the field. These properties are similar to the characteristics of the electromagnetic-waves propagation in geometrical optics (i.e., for monochromatic waves, with the approximation that length wave is much smaller than obstacles and without considering reflections or diffractions).

The fast marching method has previously been used in path planning by Sethian [35], [36] but using only an attractive potential. This method has some problems. The most important one that typically arises in mobile robotics is that optimal motion plans may bring robots very close to obstacles (including people), which is not safe. This problem has been dealt with by Latombe [14], and the resulting navigation function is called NF2. The Voronoi method also tries to follow a maximum clearance map [6]. Melchior et al. [23], [28] present a fractional potential to diminish the obstacle danger level and improve the smoothness of the trajectories, Philippsen and Siegwart [27] introduces an interpolated navigation function but with trajectories very close to obstacles and without smooth properties, and Petres et al. [26] introduce efficient path-planning algorithms for underwater vehicles that take advantage of underwater currents.
LaValle [15] deals with on-the-feedback motion-planning concept. When moving in the physical world, actions must be planned, depending on the information gathered during execution.

Lindemann and LaValle [17], [18] present a method in which the vector field globally solves the navigation problem and provides robustness to disturbances in sensing and control. In addition to being globally convergent, the vector field's integral curves (system trajectories) are guaranteed to avoid obstacles and are $\mathcal{C}^{\infty}$ smooth, except in the changes of cells. They construct a vector field with these properties by using existing geometric algorithms to partition the space into simple cells; they then define local vector fields for each cell and smoothly interpolate between them to obtain a global vector field that can be used as a feedback control for the robot.

Yang and LaValle [41] presented randomized framework motion strategies by defining a global navigation function over a collection of spherical balls in the configuration space. Their key idea is to fill the collision-free subset of the configuration space with overlapping spherical balls and define collisionfree potential functions on each ball. A similar idea has been developed for collision detection in [31] and [32].

The proposed method constructs a vectorial field as in the work of Lindemann, but the field is done in the global map instead of having local cells maps with the problem of having trajectories that are not $\mathcal{C}^{\infty}$ in the union between cells. The method also has similarities with the Yang and LaValle method. They proposed a series of balls with a Lyapunov potential associated with each of them. These potentials are connected in such a way that it is possible to find the trajectory using the gradient method in each ball. The method that we propose has a unique global Lyapunov potential associated with the vectorial field that allows for building the $\mathcal{C}^{\infty}$ trajectory in a single pass with the gradient method.

To achieve a smooth safe path, it is necessary to have smooth attractive repulsive potentials, which are connected in such a way that the resulting potential and the trajectories have no local minima and curvature continuity to facilitate the path-tracking design. The main improvement of the proposed method includes the good properties of smoothness and the safety of the trajectory. Moreover, the associated vector field allows the introduction of nonholonomic constraints.

Note that, in the proposed method, the important ingredients are the attractive and the repulsive potentials, i.e., the manner of connecting them, which describes the attractive potential using the wave equation (or in a simplified way, the eikonal equation). This equation can be solved in other ways. Mauch [22] uses a marching-with-correctness criterion with a computational complexity that can be reduced to $\mathcal{O}(N)$. Covello and Rodrigue [1] present a method that can be used on nodes that are on highly distorted grids or are randomly located.

\section{VFM MOTION PlanNer}

Which properties and characteristics are desirable for a motion planner of a mobile robot? The first one is that the planner always drives the robot in a smooth safe way to the goal point. In nature, there are phenomena with the same way of working, 

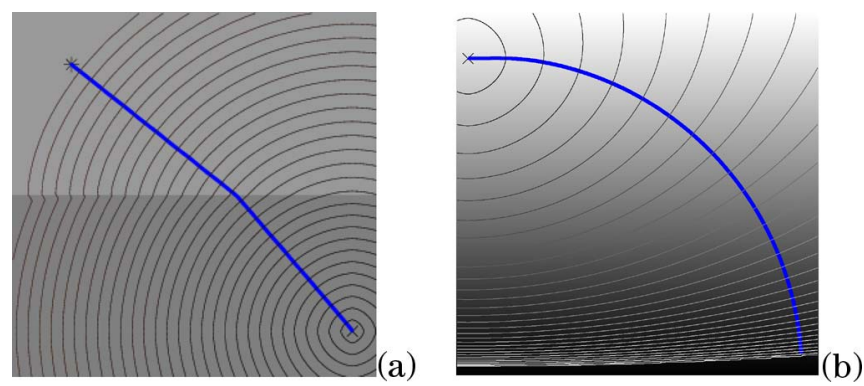

Fig. 1. Propagation of a wave and the corresponding minimum time path when there are two media of different slowness (diffraction) index. (a) With a vertical gradient. (b) Wavefront that propagates with velocity $F$.

i.e., electromagnetic waves. If, in the goal point, there is an antenna that emits an electromagnetic wave, then the robot could drive itself to the destination following the waves to the source. The concept of the electromagnetic wave is particularly interesting, because the potential and its associated vector field have all the good properties desired for the trajectory, e.g., smoothness (it is $C^{\infty}$ ) and the absence of local minima. This attractive potential still has some problems. The most important one that typically arises in mobile robotics is that optimal motion plans may bring robots very close to obstacles, which is not safe. This problem has been dealt with by Latombe [14], and the resulting navigation function is called NF2. The Voronoi method also tries to follow a maximum clearance map [5]. To generate a safe path, it is necessary to add a component that repels the robot away from obstacles. In addition, this repulsive potential and its associated vector field should have good properties such as those of the electrical field. If we consider that the robot has an electrical charge of the same sign as the obstacles, then the robot would be pushed away from obstacles. The properties of this electric field are very good, because it is smooth, and there are no singular points in the interest space $C_{\text {free }}$.

The third part of the problem consists of how the two fields can be mixed together. This union between an attractive and a repulsive field has been the biggest problem for the potential fields in path planning since the works of Khatib and Chatila [11]. In the VFM method, this problem has been solved in the same way that nature does: electromagnetic waves, e.g., light, have a propagation velocity that depends on the media. For example, flint glass has a refraction index of 1.6, whereas in the air, it is approximately 1 . This refraction index of a medium is the quotient between the velocity of light in the vacuum and the velocity in the medium under consideration. That value is the slowness index of the front-wave propagation in a medium. A light ray follows a straight line if the medium has a constant refraction index (the medium is homogeneous) but refracts when there is a transition of medium (a sudden change in the refraction index value), as shown in Fig. 1(a). In the case of a gradient change in refraction index in a given medium, the light ray follows a curved line, as shown in Fig. 1(b). This phenomenon is shown in nature as hot road mirages. In this phenomenon, the air closer to the road surface is warmer than the higher level layers. The warmer air has lower density and lower refraction index. Thus, light rays that come from the sun are curved near the road surface and cause what is called the hot

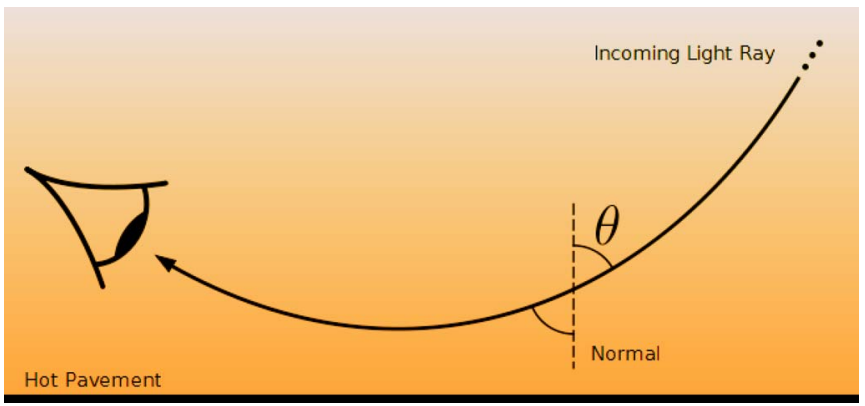

Fig. 2. Light rays that bend due to changing refraction index in the air with higher temperature near the road surface.

road mirage, as illustrated in Fig. 2. This case is the idea that inspires the way in which the attractive and the repulsive fields are merged in our work.

For this reason, in the VFM method, the repulsive potential is used as refraction index of the wave emitted from the goal point. This way, a unique field is obtained, and its associated vector field is attractive to the goal point and repulsive from the obstacles. This method inherits the properties of the electromagnetic field. Intuitively, the VFM method gives the propagation of a front wave in an inhomogeneous media, as shown in Fig. 1(b).

In geometrical optics, Fermat's least time principle for light propagation in a medium with a space-varying refractive index $\eta(\mathbf{x})$ is equivalent to the eikonal equation and can be written as $\|\nabla \Phi(\mathbf{x})\|=\eta(\mathbf{x})$, where the eikonal $\Phi(\mathbf{x})$ is a scalar function whose isolevel contours are normal to the light rays. This equation is also known as the Fundamental Equation of the Geometrical Optics.

The eikonal (from the Greek "eikon," which means "image") is the phase function in a situation for which the phase and the amplitude are slowly varying functions of position. Constant values of the eikonal represent surfaces of constant phase or wavefronts. The normals to these surfaces are rays (the paths of energy flux). Thus, the eikonal equation provides a method for "ray tracing" in a medium of slowly varying refractive index (or the equivalent for other kinds of waves).

The theory and the numerical techniques known as fast marching methods are derived from an exposition to describe the movement of interfaces based on a resolution of the equations on partial differential equations as a boundary condition problem. The fast marching method has previously been used in path planning by Sethian [35], [36] but using only an attractive potential.

The use of the fast marching method over a slowness (refraction or inverse of velocity) potential considerably improves the quality of the calculated trajectory. On one hand, the trajectories tend to go close to the Voronoi skeleton because of the optimal conditions of this area for robot motion [5]. On the other hand, the trajectories are also considerably smooth. For a small easy L-shaped environment, the funnel-shaped potential given by the wave propagation is shown in Fig. 3(a), and the trajectory calculated by the gradient method is shown in Fig. 3(b).

The solution of the eikonal equation used in the VFM method is given by the solution of the wave equation. We have

$$
\phi=\phi_{0} e^{i k_{0}\left(\eta x-c_{0} t\right)} .
$$




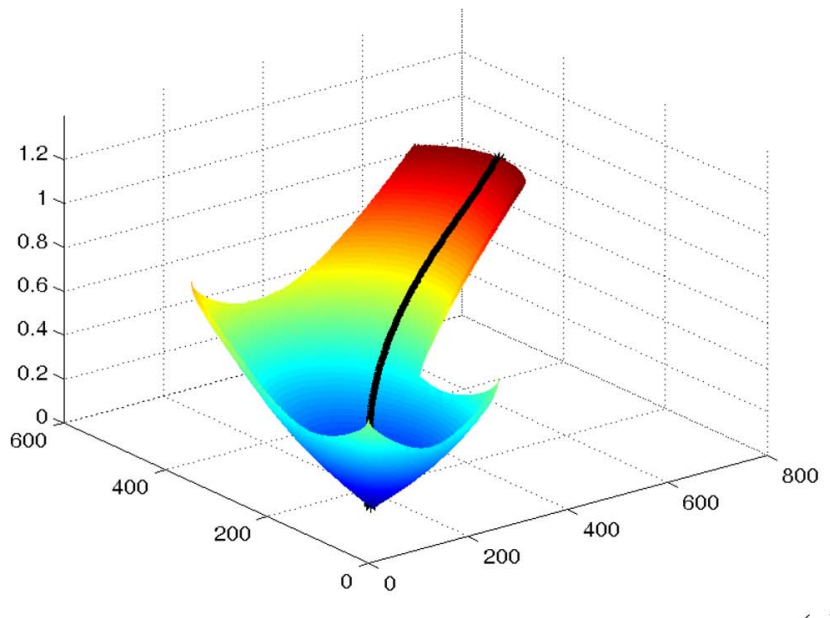

(a)

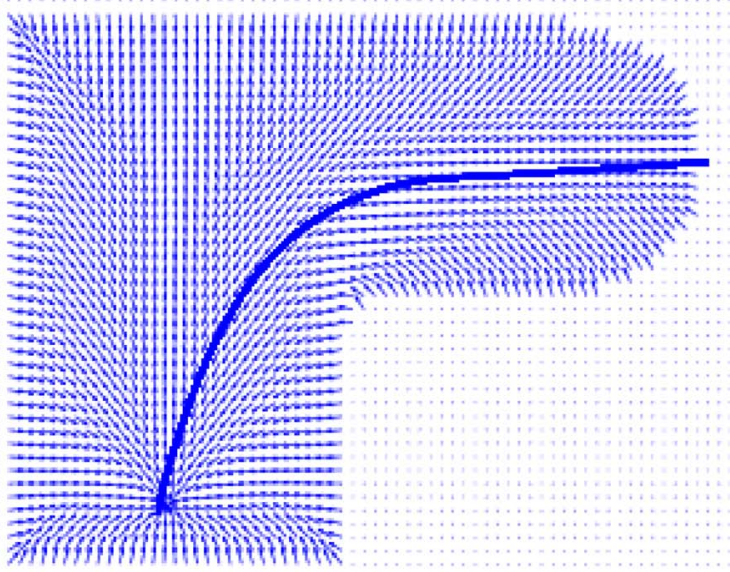

(b)

Fig. 3. (a) Union of the two potentials, with the second potential having the first one as the refractive index. (b) Associated vector field and typical trajectories obtained with this method.

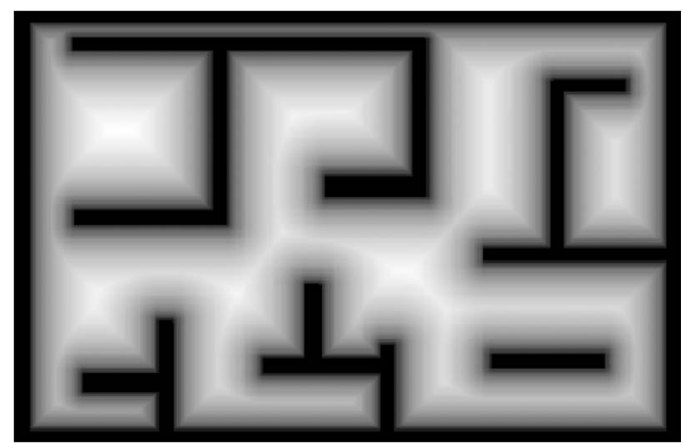

Fig. 4. Potential of the logarithm of the inverse of the EVT.

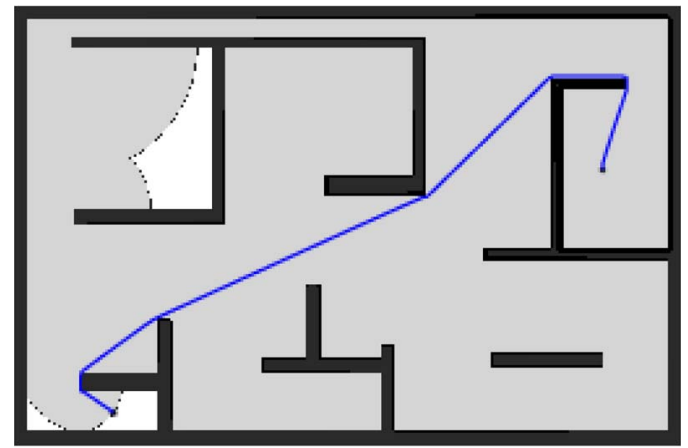

Fig. 5. Trajectory obtained by directly applying the fast marching method over the binary image without using our algorithm.

This solution is an exponential; thus, if the potential $\eta(x)$ is $\mathcal{C}^{\infty}$, then the potential $\phi$ is also $\mathcal{C}^{\infty}$, and therefore, the trajectories calculated by the gradient method over this potential would be of the same class.

This smoothness property can be observed in Fig. 6, where the trajectory is clearly good, safe, and smooth. One advantage of the method is that it generates not only the optimum path but also the velocity of the robot at each point of the path. The velocity reaches its highest values in the light areas and its minimum values in the grayer zones. The VFM method simultaneously provides the path and maximum allowable velocity for a mobile robot between the current location and the goal.

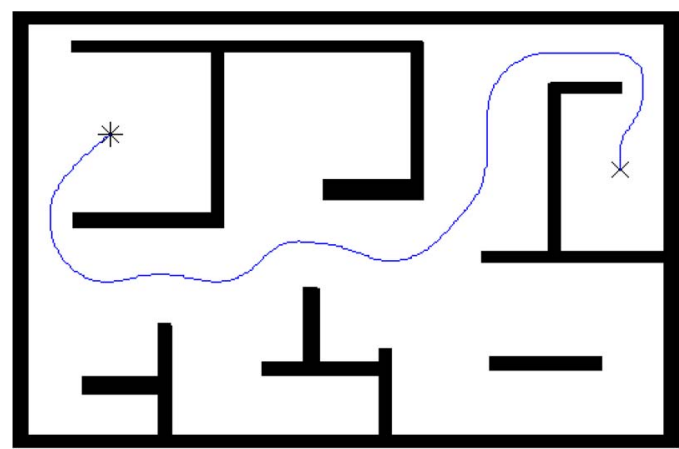

Fig. 6. Trajectories calculated by applying the proposed algorithm with fast marching over the logarithm of the EVT.

TABLE I

COMPUTATIONAL COST (IN SECONDS) FOR THE RoOM ENVIRONMENT IN FIG. $12(966 \times 120$ PIXELS $)$

\begin{tabular}{|c||c|c|c|}
\hline Alg. Step/Trajectory length & Long & Medium & Short \\
\hline Obst. Enlarging & 0.008 & 0.008 & 0.008 \\
Ext. Voronoi Transf. & 0.039 & 0.039 & 0.039 \\
FM Exploration & 0.172 & 0.078 & 0.031 \\
Path Extraction & 0.125 & 0.065 & 0.035 \\
\hline Total time & 0.344 & 0.190 & 0.113 \\
\hline
\end{tabular}

\section{A. Details of the VFM Algorithm}

This method starts with the calculation of the logarithm of the EVT of the 2-D map of the environment (or the EVT in case of 3-D maps). Each white point of the initial image (which represents free cells in the map) is associated with a level of gray that is the logarithm of the 2-D distance to the nearest obstacles (or the EVT in 3-D). As a result of this process, one kind of potential that is proportional to the distance to the nearest obstacles to each cell is obtained (see Fig. 4). Zero potential indicates that a given cell is part of an obstacle, and maxima potential cells correspond to cells in the Voronoi diagrams (which are the cells located equidistant to the obstacles).

This function introduces a potential similar to a repulsive electric potential (in 2-D), as shown in Fig. 4, which can be 
A first goal point is done

1.- Activates and reads the laser scanner.

2.- Performs update of the obstacles and walls map.

3.- Updates the Extended Voronoi Transform potential over the potential region.

4.- Calculates the Fast Marching potential at the current position.

5.- Moves following the direction defined by the gradient descent on the Fast Marching potential.

6.- Repeats this procedure until the goal point is reached.

A new goal point is done

Fig. 7. Flowchart of Case 1

expressed by

$$
\phi=c_{1} \log (r)+c_{2}
$$

where $c_{1}$ is a negative constant.

If $n>2$ (with $n$ being the space dimension), the potential is

$$
\phi=\frac{c_{3}}{r^{n-1}}+c_{4}
$$

where $r$ is the distance from the origin.

In the second step, the technique proposed here uses fast marching to calculate the shortest trajectory in the potential surface defined by the logarithm of the EVT. The calculated trajectory is the geodesic one in the potential surface, i.e., with a viscous distance. This viscosity is done by the gray level. If the fast marching method were directly used on the environment map, we would obtain the shortest geometrical trajectory, as shown in Fig. 5, but the trajectory is neither safe nor smooth.

The potential created has local minima, but the trajectories are not stuck in these points, because the fast marching method gives the trajectories that correspond to the propagation of a wave front, which is faster in lighter regions and slower in the darker ones.

The trajectories obtained using the logarithm of the EVT tend to go by the Voronoi diagram but are properly smoothed, as shown in Fig. 6.

\section{B. Properties}

The proposed VFM algorithm has the following key properties.

- Fast response. The planner needs to be fast enough to reactively be used and plan new trajectories. To obtain this fast response, a fast planning algorithm and a fast simple treatment of the sensor information are necessary. This condition requires a low-complexity-order algorithm for a real-time response to unexpected situations. The proposed algorithm has a fast response time to allow its implementation in real time, even in environments with moving obstacles using a normal PC computer.

The proposed method is highly efficient from a computational point of view, because the method directly oper- ates over a 2-D image map (without extracting adjacency maps) and due to the fact that the fast marching complexity is $O(m \times n)$ and the EVT is also of complexity $O(m \times n)$, where $m \times n$ is the number of cells in the environment map. In Table I, orientative results of the cost average in time appear (measured in seconds), and each step of the algorithm for different trajectory lengths is calculated (the computational cost depends on the number of points of the image).

- Smooth trajectories. The planner must provide a smooth motion plan that can be executed by the robot motion controller. In other words, the plan does not need to be refined, avoiding the need for a local refinement of the trajectory. The solution of the eikonal equation used in the proposed method is given by the solution of the wave equation. We have

$$
\phi=\phi_{0} e^{i k_{0}\left(\eta x-c_{0} t\right)} .
$$

This solution is an exponential; thus, if the potential $\eta(x)$ is $\mathcal{C}^{\infty}$, then the potential $\phi$ is also $\mathcal{C}^{\infty}$, and therefore, the trajectories calculated by the gradient method over this potential would be of the same class.

This smoothness property can be observed in Fig. 6, where the trajectory is clearly good, safe, and smooth. One advantage of the method is that it generates not only the optimum path but also the velocity of the robot at each point of the path. The velocity reaches its highest values in the light areas and its minimum values in the grayer zones. The VFM method simultaneously provides the path and the maximum allowable velocity for a mobile robot between the current location and the goal.

- Reliable trajectories. The proposed planner provides a safe (reasonably far from detected obstacles) and reliable trajectory (free from local traps). This result is due to the refraction index, which causes higher velocities far from obstacles.

- Completeness. The method consists of the propagation of a wave; thus, if there is a path from the initial position to the objective, the method can find it. 


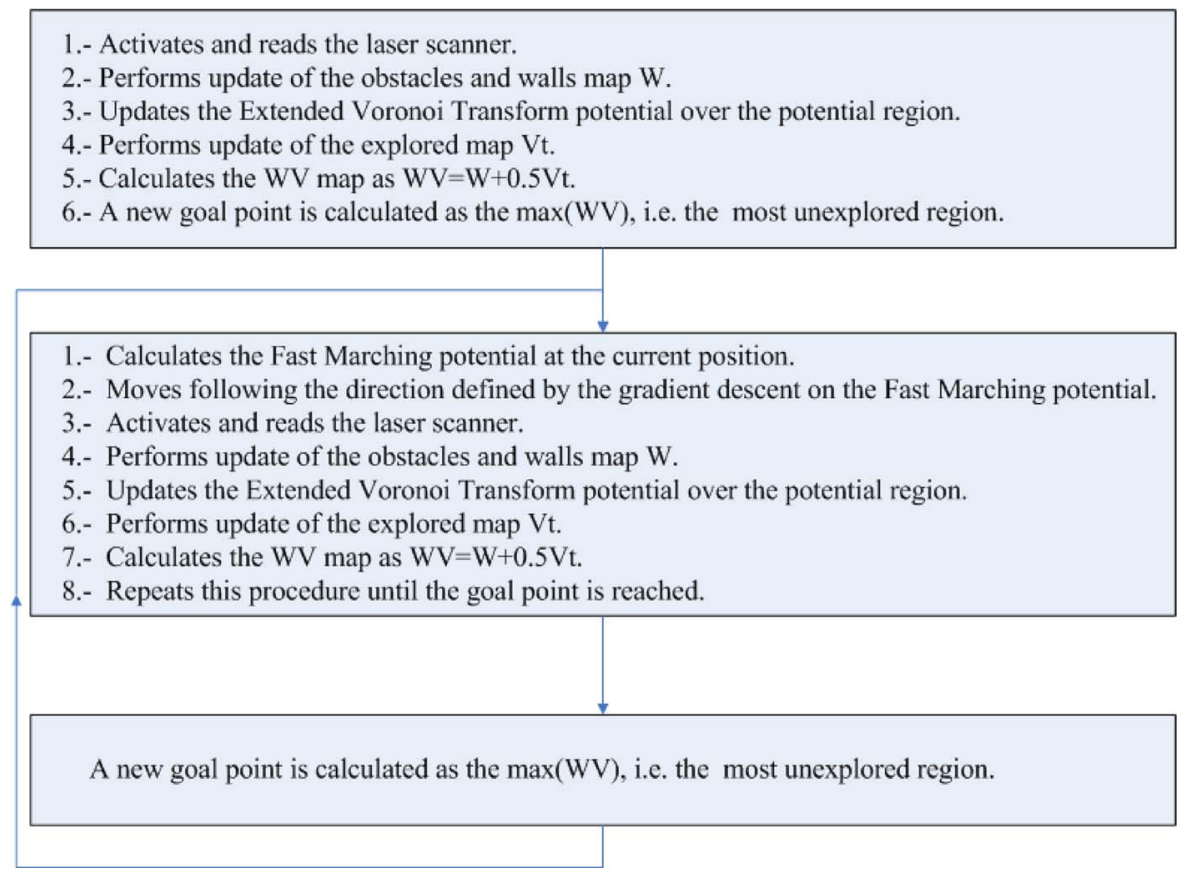

Fig. 8. Flowchart of Case 2.

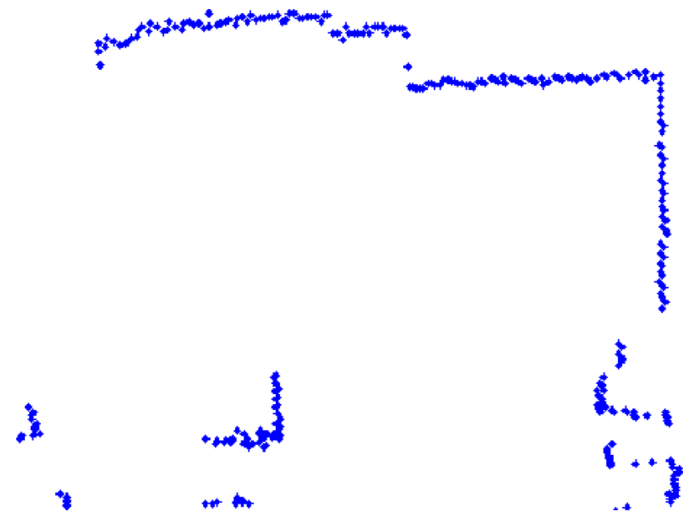

Fig. 9. Laser data read by the robot.

\section{Differential Evolution ApProach to Slam}

Localization and map building are key components in robot navigation and are required to successfully execute the path generated by the VFM planner in the exploration method proposed in this paper. Both problems are closely linked, and learning maps are required to simultaneously solve both problems - this case is the SLAM problem. Uncertainty in sensor measures and uncertainty in robot pose estimates make use of a SLAM method necessary to create a consistent map of the explored environment.

The SLAM algorithm used in this paper is described in [2020]. It is based on the stochastic search of solutions in the state space to the localization problem through a differential evolution algorithm. A nonlinear evolutive filter, called ELF, stochastically searches along the state space for the best robot pose estimate. The proposed SLAM algorithm operates in two steps. In the first step, the ELF filter is used at a local level to relocalize the robot based on the robot odometry, the laser scan at a given position, and a local map where only a low number

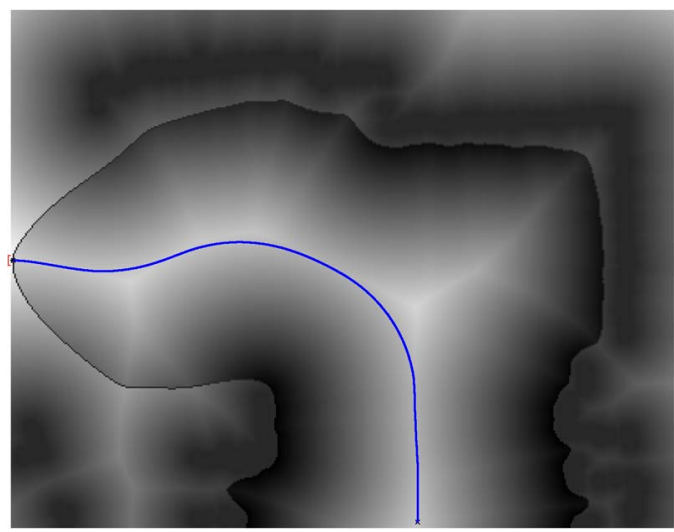

Fig. 10. Trajectory calculated with the VFM method using the laser data (local map).

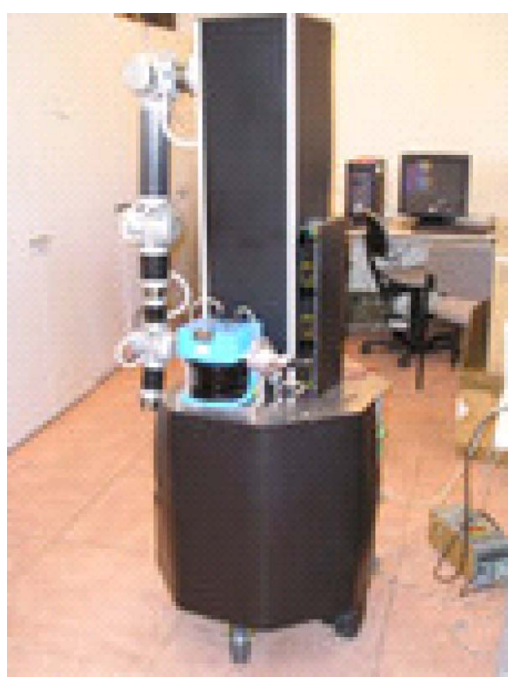

Fig. 11. Robot Manfred has been used to test the algorithms. 


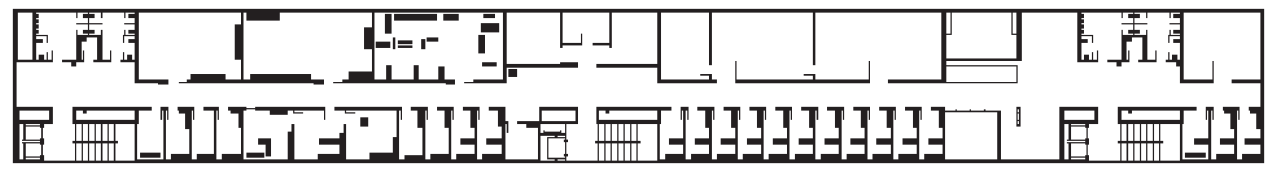

Fig. 12. Environment map of the Robotics Laboratory.

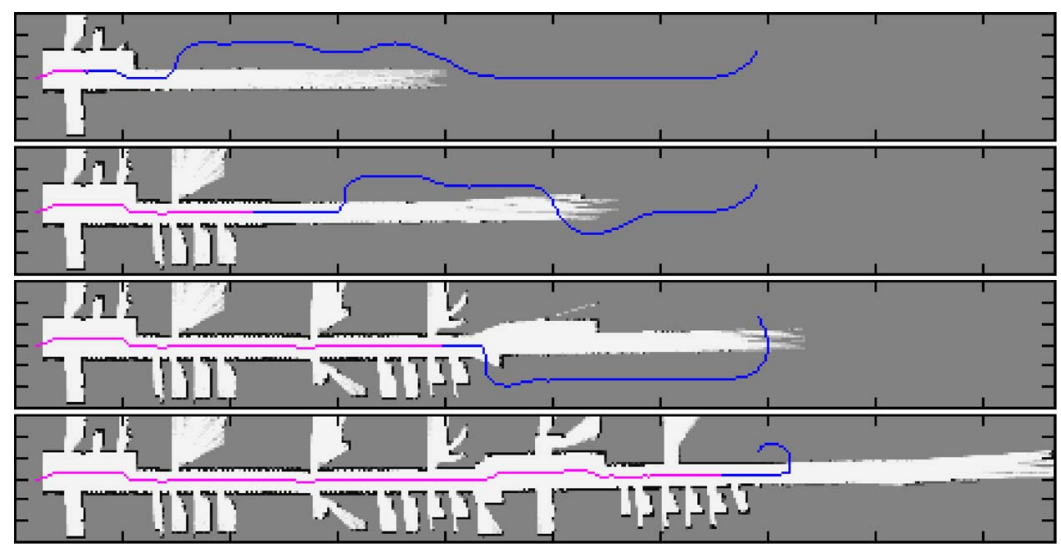

Fig. 13. Consecutive steps of the process using the first case of the exploration algorithm. The red line represents the crossed path, and the blue one represents the calculated trajectory from the present position to the destination point.

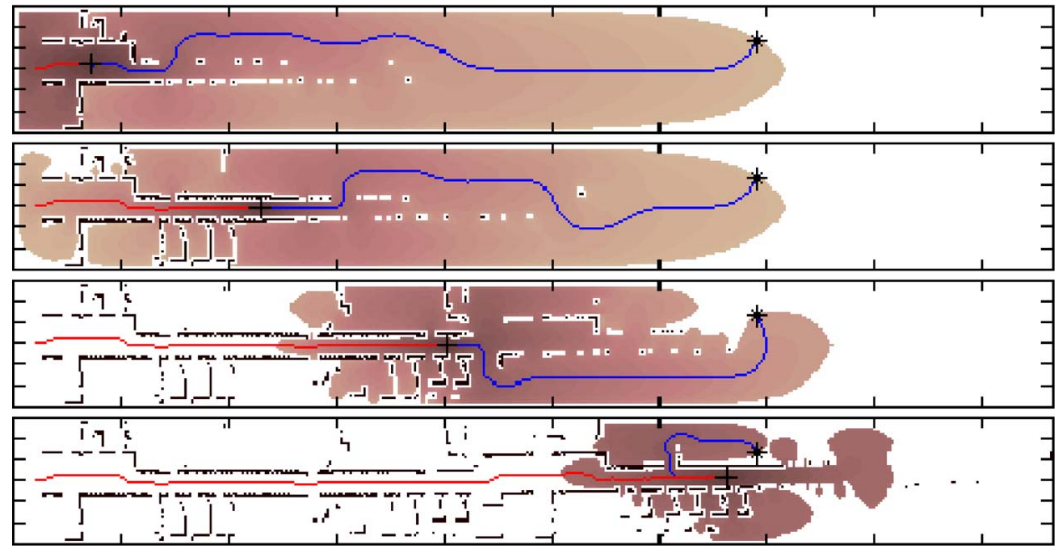

Fig. 14. Consecutive steps of the process. The illuminated area represents the front-wave propagation.
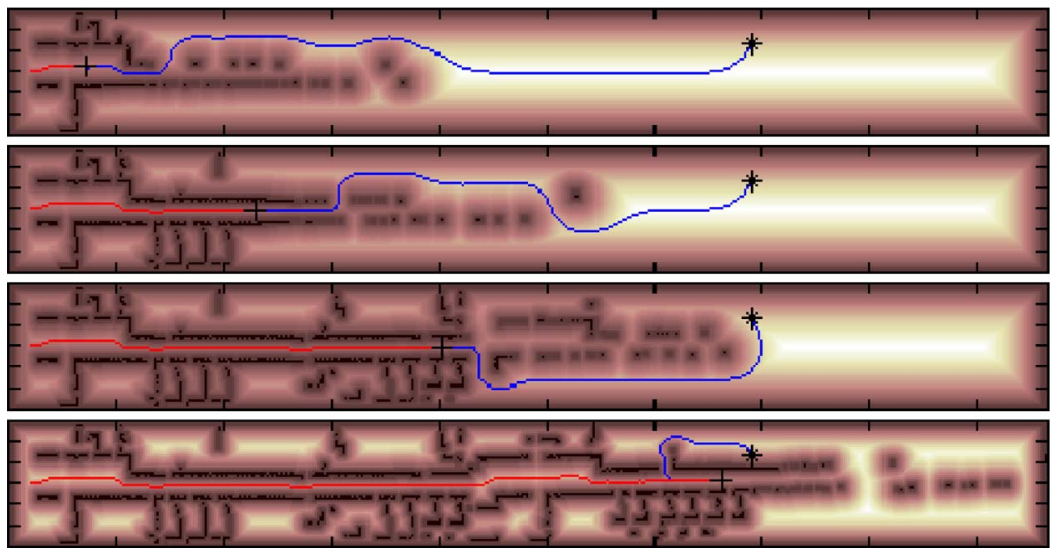

Fig. 15. Map built in each step using the SLAM algorithm.

of the last scans have been integrated. In the second step, the aligned laser measures, together with the corrected robot poses, are used to detect when the robot revisits a previously crossed area. Once a cycle is detected, the ELF is reused to reestimate the robot position and orientation to integrate the sensor measures in the global map of the environment.
This approach uses a differential evolution method to perturb the possible pose estimates contained in a given set until the optimum is obtained. By properly choosing the cost function, a maximum a posteriori estimate is obtained. This method is applied at a local level to relocalize the robot and at a global level to solve the data association problem. The method 

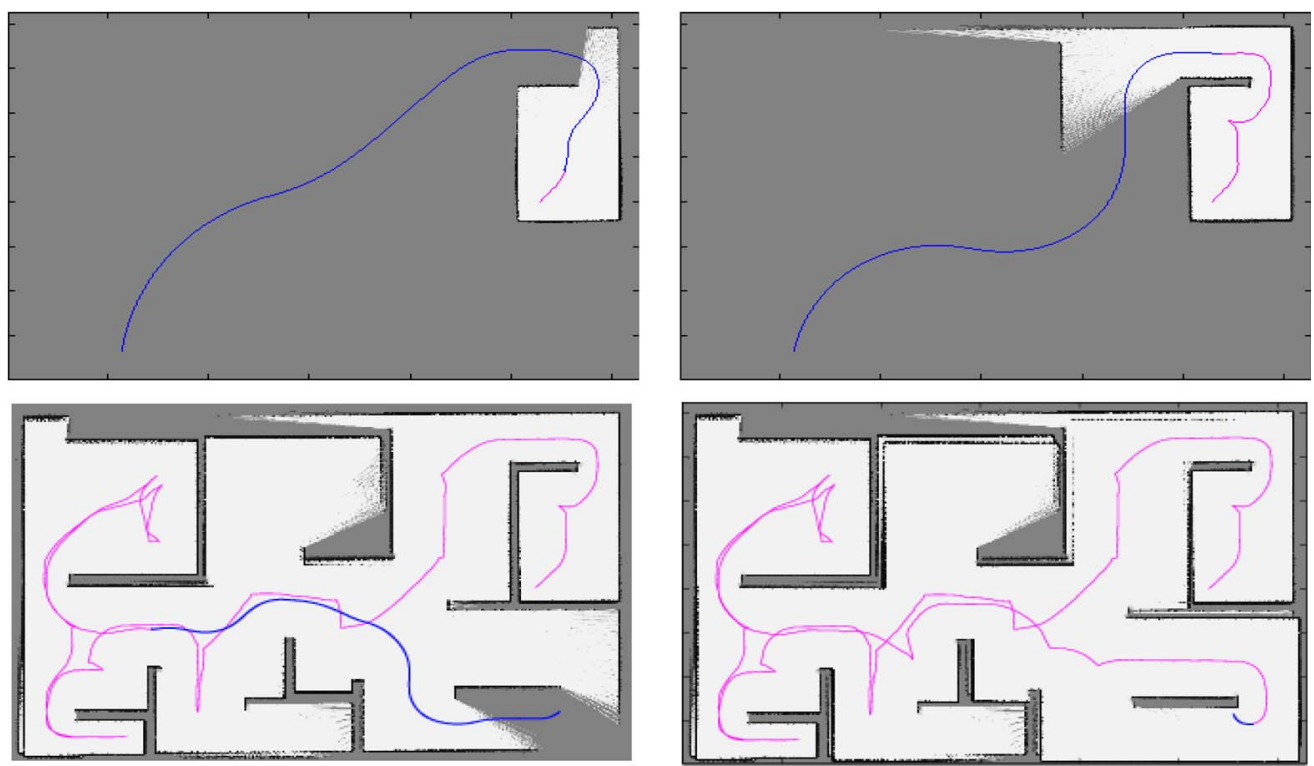

Fig. 16. Simulation results with Case 1, with the final objective. Trajectory was calculated. The red line represents the crossed path, and the blue one represents the calculated trajectory from the present position to the destination point.
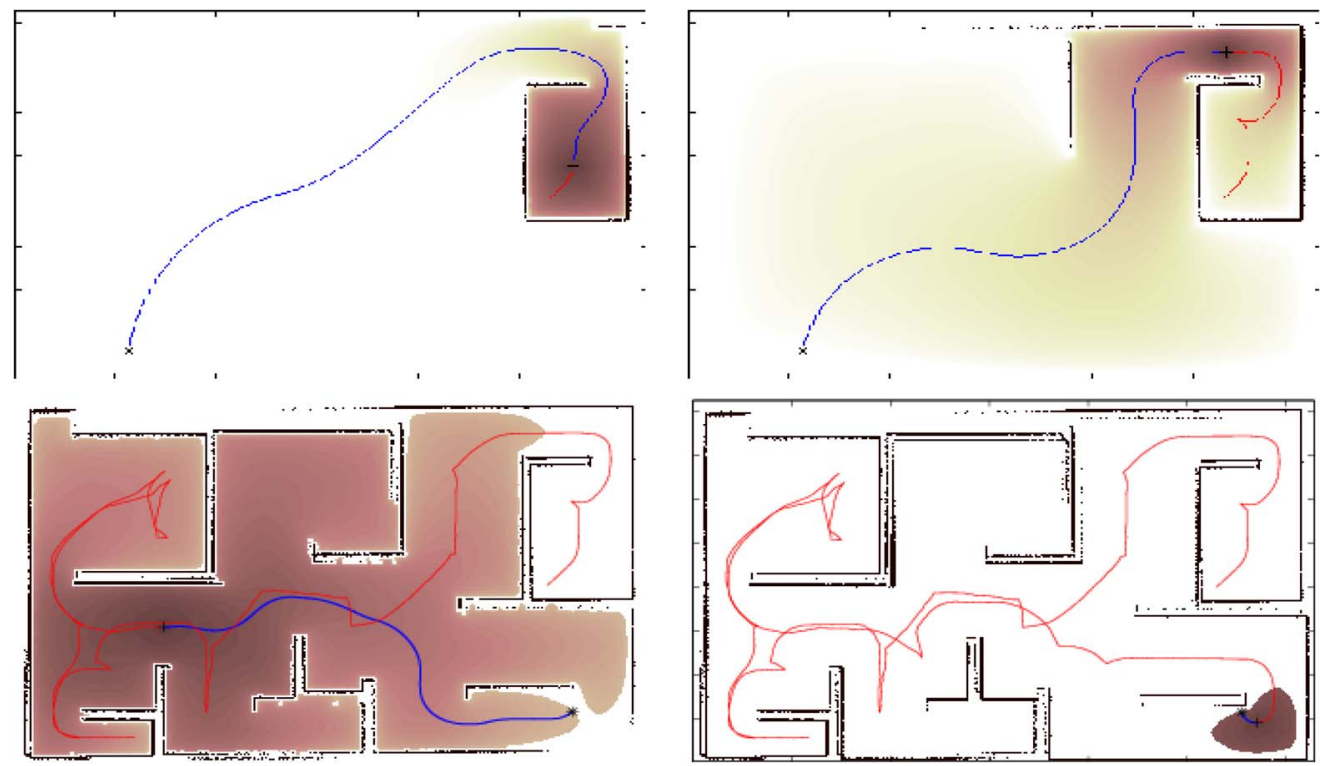

Fig. 17. Simulation results with Case 1 with front-wave expansion. The red line represents the crossed path, and the blue one represents the calculated trajectory from the present position to the destination point.

proposed integrates sensor information in the map only when cycles are detected, and the residual errors are eliminated, thus avoiding a large number of modifications in the map or the existence of multiple maps and decreasing the computational cost compared to other solutions.

\section{IMPLEMENTATION OF THE EXPLORER}

To solve the problem of the exploration of an unknown environment, our algorithm can work in two different ways. First, the exploration process can be directed by giving the algorithm one or several successive goal points in the environment to which the robot must drive during exploration. Second, which is the second form of our algorithm, the exploration can be carried out without having any previously fixed objective point. In such a case, the algorithm must automatically determine toward where the robot must drive to complete the exploration process.

Case I: In the first case, the initial information is the localization of the final goal. This way, the robot has a general direction of movement toward the goal. In each movement of the robot, information about the environment is used to build a binary image that distinguishes the occupied space represented by value 0 (obstacles and walls) from the free space, with value 1 . The EVT of the known map at that moment gives a gray scale that is darker near the obstacles and walls and is lighter far from them. The VFM method gives the trajectory from the pose of the robot to the goal point using the known information. In this first way of working, the SLAM algorithm in [2020] is used to avoid localization errors from being translated into the map built during exploration. 

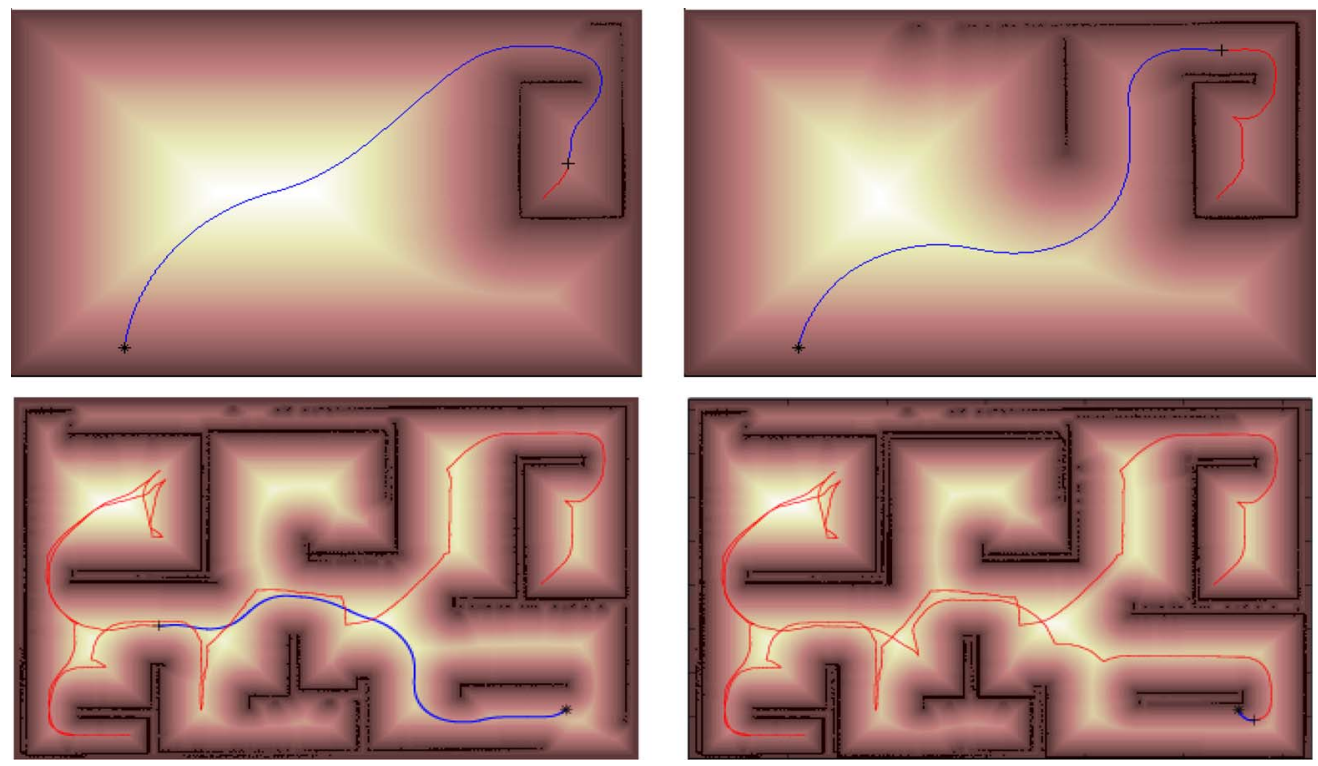

Fig. 18. Simulation results with Case 1 with the map built. The red line represents the crossed path, and the blue one represents the calculated trajectory from the present position to the destination point.

In this first case, the robot has a final goal: in the exploration process, the robot performs the algorithm in the flowchart in Fig. 7.

Case II: In the second form of the algorithm, the goal location is unknown, and the robot behavior is truly exploratory. We propose an approach based on the incremental calculation of a map for path planning.

We define a neighborhood window, which travels with the robot, roughly the size of its laser sensor range. This window indicates the new grid cells that are recruited for update; i.e., if a cell was in the neighborhood window at a given time, it becomes part of the explored space by participating in the EVT and fast marching method calculation for all times. The set of activated cells that comprise the explored space is called the neighborhood region. Cells that were never inside the neighborhood window indicate unexplored regions. Their potential values are set to zero and define the knowledge frontier of the state space, i.e., the real space in our case. The detection of the nearest unexplored frontier naturally comes from the EVT calculation. It can also be understood from the physical analogy with electrical potentials that obstacles repel, whereas frontiers attract.

Consider that the robot starts from a given position in an initially unknown environment. In this second method, there is no direction of the place where the robot must go.

An initial matrix with zeros in the obstacles and value 1 in the free zones is considered. This first matrix is built using the information provided by sensors and represents a binary image of the environment detected by sensors. The first step consists of calculating the EVT of the obstacles in this image. A value that represents the distance to the nearest obstacle is associated with each cell of the matrix. A matrix $W$ of grays with values between 0 (obstacles) and 1 is obtained. This $W$ matrix gives us the EVT of the obstacles found up until that moment.

The second matrix is built by darkening the zones that the robot has already visited. Then, the EVT of this image is calculated, and the result is the $V T$ matrix.

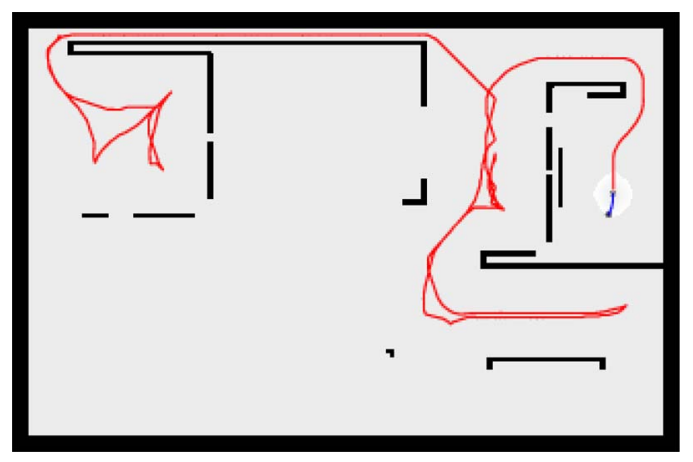

Fig. 19. Trajectory followed to explore an unknown environment with the method proposed by the first algorithm.

Finally, matrix $W V$ is the sum of the matrices $V T$ and $W$, with weights 0.5 and 1 , respectively, i.e.,

$$
W V=0.5 * V T+W .
$$

This way, it is possible to darken the zones already visited by the robot and impel it to go to the unexplored zones. The whitest point of matrix $W V$ is calculated as $\max (W V)$, i.e., the most unexplored region that is in a free space. This point is chosen as the new goal point. Applying the fast marching method on $W V$, the trajectory toward that goal is calculated. The robot moves by following this trajectory. In the following steps, the trajectory to be followed is computed, first calculating $W$ and $V T$ at every moment and, therefore, $W V$ but without changing the objective point. Once the robot has been arrived at the objective (i.e., that path calculated is very small), a new objective is selected as $\max (W V)$.

Therefore, the robot moves by maximizing the knowledge gain. In this case or in any other situation where there is no gradient to guide the robot, it simply follows the forward direction. The exploration process that the robot performs in the second method is summarized in the flowchart in Fig. 8. 

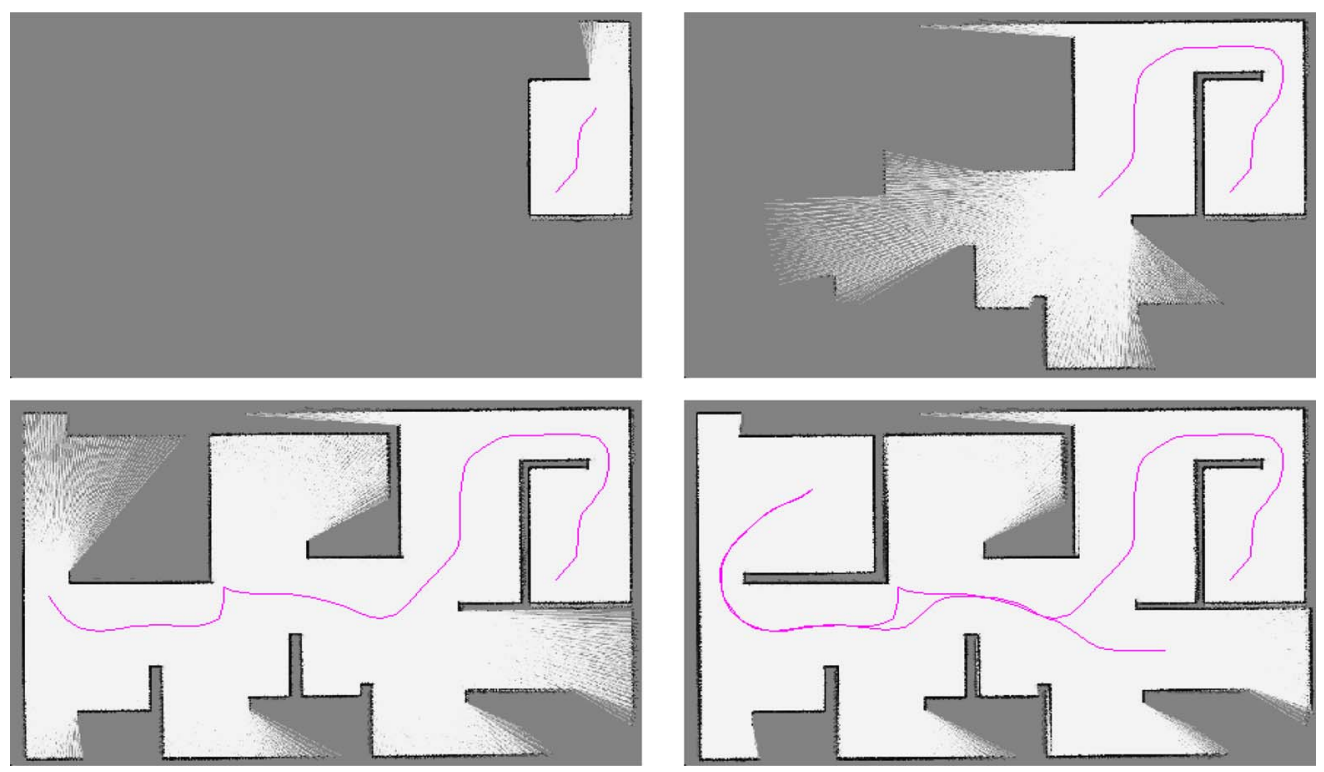

Fig. 20. Simulation results with Case 2, without the final objective. Trajectory was calculated.

The algorithms laid out in Fig. 7 (i.e., the flowchart of Case 1) can be inefficient in very large environments. To increase the speed, it is possible to pick a goal point, put a neighborhood window the size of the sensor range, run into the goal point, then look at the maximal initial boundary, and recast and terminate when one reaches the boundary of the computed region. Similar improvements can be made to Case 2 .

\section{RESULTS}

The proposed method has been tested using the manipulator robot Manfred (see Fig. 11). It has a coordinated control of all degrees of freedom in the system (the mobile base has 2 DOFs, and the manipulator has 6 DOFs) to achieve smooth movement. This mobile manipulator uses a sensorial system based on vision and 3-D laser telemetry to perceive and model 3-D environments. The mobile manipulator will include all the capabilities needed to safely navigate, localize, and avoid obstacles throughout the environment.

The VFM method is used for sensor-based planning, directly working on a raw sensor image of the environment, as shown in Figs. 9 and 10. These images correspond to a corner of a corridor of our University.

To illustrate the performance of the exploration method based on the VFM motion planner proposed, a test (see Fig. 11) in a typical office indoor environment, as shown in Fig. 12, has been carried out. The dimensions of the environment are $116 \times 14 \mathrm{~m}$ (the cell resolution is $12 \mathrm{~cm}$ ); i.e., the image has $966 \times 120$ pixels.

The VFM motion-planning method provides smooth trajectories that can be used at low control levels without any additional smooth interpolation process. Some of the steps of the planning process between two defined points are shown in Figs. 13-15. In Fig. 13, the trajectory computed by the VFM planner is represented (the red line represents the crossed path, and the blue one represents the calculated trajectory from the present position to the destination point). In each step, the illu- minated area in Fig. 14 represents the front-wave propagation from the present position of the robot to the destination point. The EVT computation is made on the sensory map that the robot has in its memory. Finally, Fig. 15 shows the map built in each step using the SLAM algorithm.

For the cases of exploration that this paper contemplates, the results of two different tests are presented to illustrate both cases described for the application of the proposed method in the same environment. In this case, the size of the image is $628 \times 412$.

Figs. 16-18 represent the first case for implementing the exploration method (directed exploration). A final goal is provided for the robot, which is located with respect to a global reference system; the starting point of the robot movement is also known with respect to that reference system. The algorithm allows for calculating the trajectory toward that final goal with the updated information of the surroundings that the sensors obtain in each step of the movement. When the robot reaches the defined goal, a new destination in an unexplored zone is defined, as shown in the third image of the figure.

Fig. 19 shows an example of the exploration without knowing that the environment applies the first algorithm. The trajectory goes from left to right, and the cusp points correspond to direction changes when the robot finds a wall that interrupts the planned trajectory. This figure shows that the algorithm can generate a trajectory through narrow passages.

The results of one of the tests for the second case of exploration are shown in Figs. 20-22. Any final goal is defined. The algorithm leads the robot toward the zones that are free of obstacles and simultaneously unexplored (undirected exploration).

\section{CONCLUSION}

The results obtained have shown that the logarithm of EVT can be used to improve the results obtained with the fast marching method to implement a sensor-based motion planner, which provides smooth and safe trajectories. 

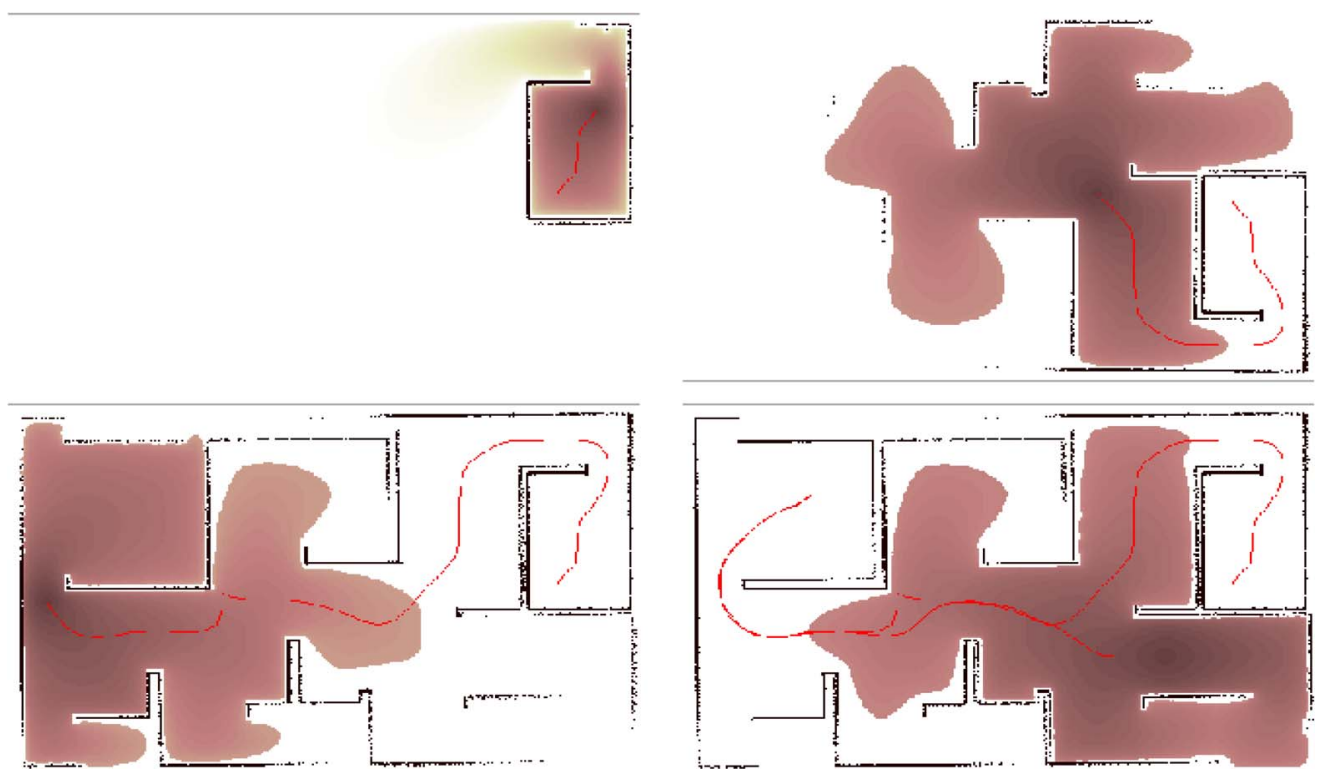

Fig. 21. Simulation results with Case 2 with front-wave expansion.
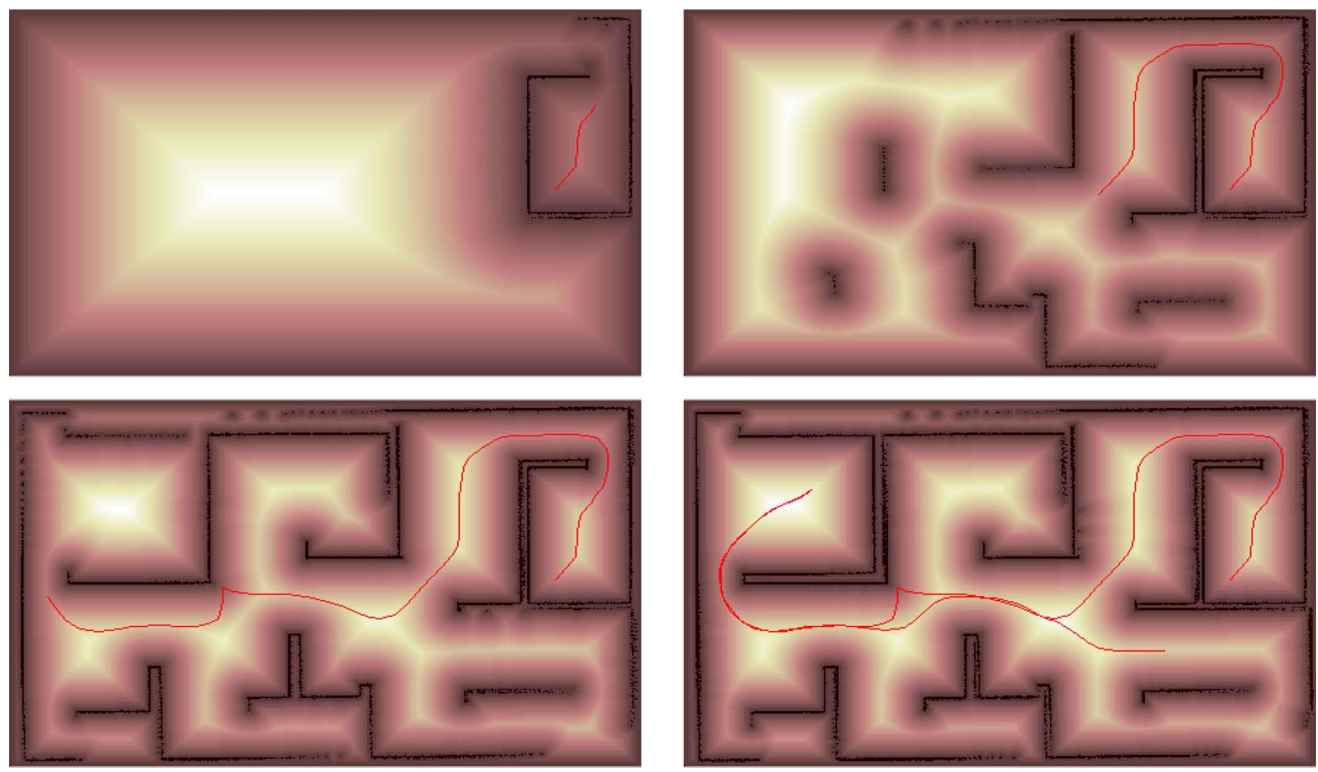

Fig. 22. Simulation results with Case 2 with the map built.

The algorithm complexity is $O(m \times n)$, where $m \times n$ is the number of cells in the environment map, which lets us use the algorithm online. Furthermore, the algorithm can directly be used with raw sensor data to implement a sensor-based localpath-planning exploratory module.

This paper has presented a new autonomous exploration strategy. The essential mechanisms used has included the VFM method [77] that plans the trajectory toward the goal, a new exploratory strategy that drives the robot to the most unexplored region, and the SLAM algorithm [2020] that builds a consistent map of the environment. The proposed autonomous exploration algorithm is a combination of the three tools that can completely construct consistent maps of unknown indoor environments in an autonomous way.

\section{REFERENCES}

[1] P. Covello and G. Rodrigue, "A generalized front marching algorithm for the solution of the eikonal equation," J. Comput. Appl. Math., vol. 156, no. 2, pp. 371-388, Jul. 2003.

[2] A. Elfes, "Sonar-based real-world mapping and navigation," IEEE J. Robot. Autom., vol. RA-3, no. 3, pp. 249-265, Jun. 1987.

[3] A. Elfes, "Using occupancy grids for mobile robot perception and navigation," Comput. Mag., vol. 22, no. 6, pp. 46-57, Jun. 1989.

[4] H. Feder, J. Leonard, and C. Smith, "Adaptive mobile robot navigation and mapping," Int. J. Robot. Res., vol. 18, no. 7, pp. 650-668, 1999.

[5] S. Garrido, L. Moreno, and D. Blanco, "Voronoi diagram and fast marching applied to path planning," in Proc. IEEE ICRA, 2006, pp. 3049-3054.

[6] S. Garrido, L. Moreno, M. Abderrahim, and F. Martin, "Path planning for mobile robot navigation using Voronoi diagram and fast marching," in Proc. IROS, Beijing, China, 2006, pp. 2376-2381.

[7] S. Garrido, L. Moreno, and D. Blanco, "Sensor-based global planning for mobile robot navigation," Robotica, vol. 25, no. 2, pp. 189-199, Mar. 2007. 
[8] A. Howard and L. Kitchen, "Generating sonar maps in highly specular environments," in Proc. 4th Int. Conf. Control, Autom., Robot. Vis., 1996, pp. 1870-1874.

[9] Y. Koren and J. Borenstein, "Histogramic in-motion mapping for mobile robot obstacle avoidance," IEEE J. Robot., vol. 7, no. 4, pp. 535-539, Aug. 1991.

[10] Y. Koren and J. Borenstein, "Potential field methods and their inherent limitations for mobile robot navigation," in Proc. IEEE Int. Conf. Robot. Autom., 1991, pp. 1398-1404.

[11] M. Khatib and R. Chatila, "An extended potential field approach for mobile robot sensor-based motions," in Intelligent Autonomous Systems $I A S-4$, U. Rembold, R. Dillmann, L. O. Hertzberger, and T. Kanade, Eds., Amsterdam, Netherlands: IOS Press, 1995, pp. 490-496. [Online]. Available: http://dbserver.laas.fr/pls/LAAS/publis. rech_doc?langage $=$ FR\&clef $=11552$

[12] B. Kuipers and T. Levitt, "Navigation and mapping in large-scale space," AI Mag., vol. 9, no. 2, pp. 25-43, Jul./Aug. 1988.

[13] B. Kuipers and Y. Byun, "A robot exploration and mapping strategy based on a semantic hierarchy of spatial representations," Robot. Auton. Syst., vol. 8, no. 1/2, pp. 47-63, Nov. 1991.

[14] J.-C. Latombe, Robot Motion Planning. Dordrecht, The Netherlands: Kluwer, 1991.

[15] S. M. LaValle, Planning Algorithms. Cambridge, U.K.: Cambridge Univ. Press, 2006.

[16] W. Lee, "Spatial semantic hierarchy for a physical robot," Ph.D. dissertation, Dept. Comput. Sci., Univ. Texas, Austin, TX, 1996.

[17] S. R. Lindemann and S. M. LaValle, "Simple and efficient algorithms for computing smooth, collision-free feedback laws," Int. J. Robot. Res., 2009. [Online]. Available: http://msl.cs.uiuc.edu/ $\sim$ slindema/papers/LinLav06c.pdf, to be published.

[18] S. R. Lindemann and S. M. LaValle, "Smooth feedback for car-like vehicles in polygonal environments," in Proc. IEEE Int. Conf. Robot. Autom., 2007, pp. 3104-3109.

[19] H. Moravec and A. Elfes, "High-resolution maps from wide-angle sonar," in Proc. IEEE Int. Conf. Robot. Autom., 1985, pp. 116-121.

[20] L. Moreno, S. Garrido, and F. Martíin, "E-SLAM solution to the gridbased localization and mapping problem," in Proc. IEEE Int. Symp. WISP, Alcala Henares, Spain, 2007, pp. 897-903.

[21] M. Mataric, "Integration of representation into goal-driven behaviorbased robots," IEEE Trans. Robot. Autom., vol. 8, no. 3, pp. 304-312, Jun. 1992.

[22] S. Mauch, "Efficient algorithms for solving static Hamilton-Jacobi equations," Ph.D. dissertation, California Inst. Technol., Pasadena, CA, 2003.

[23] P. Melchior, B. Orsoni, O. Laviale, A. Poty, and A. Oustaloup, "Consideration of obstacle danger level in path planning using $\mathrm{a}^{*}$ and fast marching optimization: Comparative study," J. Signal Process., vol. 83, no. 11, pp. 2387-2396, Nov. 2003.

[24] G. Oriolo, G. Ulivi, and M. Vendittelli, "On-line map-building and navigation for autonomous mobile robots," in Proc. IEEE Int. Conf. Robot. Autom., 1995, pp. 2900-2906.

[25] G. Oriolo, G. Ulivi, and M. Vendittelli, "Fuzzy maps: A new tool for mobile robot perception and planning," J. Robot. Syst., vol. 14, no. 3, pp. 179-197, Mar. 1997.

[26] C. Petres, Y. Pailhas, P. Patron, Y. Petillot, J. Evans, and D. Lane, "Path planning for autonomous underwater vehicles," IEEE Trans. Robot., vol. 23, no. 2, pp. 331-341, Apr. 2007.

[27] R. Philippsen and R. Siegwart, "An interpolated dynamic navigation function," in Proc. IEEE Int. Conf. Robot. Autom., 2005, pp. 3782-3789.

[28] A. Poty, P. Melchior, and A. Oustaloup, "Dynamic path planning by fractional potential," in Proc. 2nd IEEE Int. Conf. Comput. Cybern., 2004, pp. 365-371.

[29] E. Prestes da Silva, P. Engel, M. Trevisan, and M. Idiart, "Exploration method using harmonic functions," Robot. Auton. Syst., vol. 40, no. 1, pp. 25-42, Jul. 2002.

[30] E. Prestes da Silva, P. Engel, M. Trevisan, and M. Idiart, "Autonomous learning architecture for environmental mapping," J. Intell. Robot. Syst., vol. 39, no. 3, pp. 243-263, Mar. 2004.

[31] S. Quinlan and O. Khatib, "Elastic bands: Connecting path planning and control," in Proc. IEEE Int. Conf. Robot Autom., 1993, pp. 802-807.

[32] S. Quinlan and O. Khatib, "Efficient distance computation between nonconvex objects," in Proc. IEEE Int. Conf. Robot Autom., 1994, pp. 3324-3329.

[33] E. Rimon and D. E. Koditschek, "Exact robot navigation using artificial potential functions," IEEE Trans. Robot. Autom., vol. 8, no. 5, pp. 501518, Oct. 1992.
[34] J. A. Sethian, "A fast marching level set method for monotonically advancing fronts," Proc. Nat. Acad. Sci. U.S.A., vol. 93, no. 4, pp. 15911595, Feb. 1996.

[35] J. A. Sethian, "Theory, algorithms, and applications of level set methods for propagating interfaces," in Acta Numer, vol. 5, 1996, pp. 309-395.

[36] J. Sethian, Level Set Methods. Cambridge, U.K.: Cambridge Univ. Press, 1996.

[37] S. Thrun and A. Bucken, "Integrating grid-based and topological maps for mobile robot," in Proc. 13th Nat. Conf. Artif. Intell. (AAAI), 1996, pp. 944-950.

[38] S. Thrun and A. Bucken, "Learning maps or indoor mobile robot navigation," Carnegie Mellon Univ., Pittsburgh, PA, Tech. Rep. CMU-CS-96121, 1996.

[39] B. Yamauchi, "A frontier-based exploration for autonomous exploration," in Proc. IEEE Int. Symp. Comput. Intell. Robot. Autom., Monterey, CA, 1997, pp. 146-151.

[40] B. Yamauchi, A. Schultz, W. Adams, and K. Graves, "Integrating map learning, localization and planning in a mobile robot," in Proc. IEEE Int. Symp. Comput. Intell. Robot. Autom., Gaithersburg, MD, 1998, pp. 331-336.

[41] L. Yang and S. M. LaValle, "A framework for planning feedback motion strategies based on a random neighborhood graph," in Proc. IEEE Int. Conf. Robot. Autom., 2000, pp. 544-549.

[42] J. Zelek, "A framework for mobile robot concurrent path planning and execution in incomplete and uncertain environments," in Proc. AIPS Workshop Integrating Planning, Scheduling Execution Dynamic Uncertain Environments, Pittsburgh, PA, 1998.

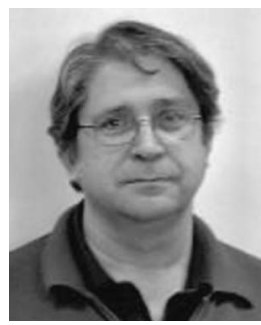

Santiago Garrido received the B.S. degree in mathematics from the Complutense University of Madrid, Madrid, Spain, and the Master's degree in physics science and the Ph.D. degree from the Universidad Carlos III de Madrid (UC3M), Madrid, in 1995 and 2000, respectively.

In 1997, he joined the Department of Systems Engineering and Automation, UC3M, where he has been involved in several mobile robotics projects. His research interests include mobile robotics, mobile manipulators, environment modeling, sensor based robot motion, and mobile robot global localization problems.

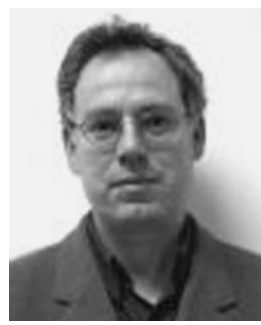

Luis Moreno received the B.S. degree in automation and electronics engineering and the Ph.D. degree from the Universidad Politécnica de Madrid, Madrid, Spain, in 1984 and 1988, respectively.

From 1988 to 1994, he was an Associate Professor with the Universidad Politécnica de Madrid. In 1994, he joined the Department of Systems Engineering and Automation, Universidad Carlos III de Madrid (UC3M), Madrid, where he has been involved in several mobile robotics projects. His research interests include mobile robotics, mobile manipulators, environment modeling, path planning, and mobile robot global localization problems.

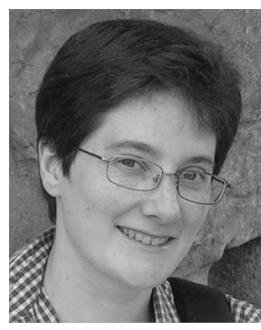

Dolores Blanco received the B.S. degree in physics from the University Complutense of Madrid, Madrid, Spain, in 1992 and the Ph.D. degree in mecatronics from the Universidad Carlos III de Madrid (UC3M), Madrid, in 2002.

From 1996 to 1999, she was a Fellowship Student with the Department of Systems Engineering and Automation, UC3M, where she has been an Assistant Professor since 1999. She is a member of the Mobile Manipulator Group, UC3M. Her research interests include sensor-based path planning, localization, and control for mobile manipulators. 